\title{
Effect of rifampicin on the Correspondence
}

Effect of rifampicin on the pharmacokinetics and

\section{pharmacodynamics of}

\section{saxagliptin, a dipeptidyl peptidase-4 inhibitor, in healthy subjects}

Vijay V. Upreti PhD, FCP, Discovery Medicine and Clinical Pharmacology, Bristol-Myers Squibb Company, Research \& Development, Route 206 \& Province Line Road, Princeton, NJ 08543, USA.

Tel.: +1 6092525699

Fax: +1 6092527821

E-mail:vijay.upreti@bms.com

Keywords

dipeptidyl peptidase-4 (DPP-4) inhibitor, rifampicin, rifampin, saxagliptin

Received

17 August 2010

Accepted

8 February 2011

Accepted Article

18 February 2011

Vijay V. Upreti, ${ }^{1}$ David W. Boulton,
Frank P. LaCreta ${ }^{1} \&$ Chirag G. Patel $^{1}$

'Discovery Medicine and Clinical Pharmacology, Bristol-Myers Squibb Company, ${ }^{2}$ Global Biometric

Sciences, Bristol-Myers Squibb Company and ${ }^{3}$ Pharmaceutical Candidate Optimization,

Biotransformation, Bristol-Myers Squibb Company, Princeton, NJ, USA

\section{WHAT IS ALREADY KNOWN ABOUT THIS SUBJECT}

- Rifampicin, an anti-tubercular antibiotic, is a potent inducer of cytochrome P450 (CYP) 3A4 enzymes. Rifampicin is also a potent inducer of some drug efflux transporters and an inhibitor of certain uptake transporter proteins. - Saxagliptin is a potent, selective dipeptidyl peptidase-4 (DPP-4) inhibitor, specifically designed for extended inhibition of the DPP-4 enzyme in the treatment of type 2 diabetes. - Saxagliptin is metabolized by CYP3A4/3A5 to its major metabolite, 5-hydroxy saxagliptin, which is also a potent inhibitor of DPP-4.

-A drug-drug interaction study of saxagliptin with an archetypal CYP3A4 inducer, rifampicin, provides essential information for clinical use of saxagliptin with respect to the need for dose adjustment when co-administered with CYP3A4 inducers.

\section{WHAT THIS STUDY ADDS}

- Rifampicin significantly reduced exposure to saxagliptin. There was no decrease in the area under the plasma concentration-time curve and slight increase in the peak plasma concentration for the active metabolite 5-hydroxy saxagliptin. The saxagliptin total active moieties exposure was slightly lower when co-administered with rifampicin. - There was no change in the maximum DPP-4 inhibition and area under the effect-time curve for DPP-4 inhibition when saxagliptin was co-administered with rifampicin. - Saxagliptin was generally safe and well tolerated when co-administered with rifampicin in this study. - The lack of clinically meaningful change of pharmacodynamic effect (plasma DPP-4 activity) of saxagliptin when co-administered with rifampicin is consistent with the observed slight reduction in systemic exposure to the total active moieties. Based on these findings, it is not necessary to adjust the saxagliptin dose when co-administered with rifampicin.

\section{AIM}

To investigate the effect of co-administration of rifampicin, a potent inducer of cytochrome P450 (CYP) 3A4 enzymes, on the pharmacokinetics (PK) and pharmacodynamics (PD) of saxagliptin and 5-hydroxy saxagliptin in healthy subjects. Saxagliptin is metabolized by CYP3A4/3A5 to 5-hydroxy saxagliptin, its major pharmacologically active metabolite.

\section{METHODS}

In a non-randomized, open label, single sequence design, 14 healthy subjects received single oral doses of saxagliptin $5 \mathrm{mg}$ with and without steady-state rifampicin ( $600 \mathrm{mg}$ once daily for 6 days). PK (saxagliptin and 5-hydroxy saxagliptin) and PD (plasma DPP-4 activity) were measured for up to $24 \mathrm{~h}$ on days 1 and 7 .

\section{RESULTS}

Concomitant administration with rifampicin resulted in 53\% (point estimate $0.47,90 \% \mathrm{Cl} 0.38,0.57$ ) and $76 \%$ (point estimate $0.24,90 \% \mathrm{Cl} 0.21,0.27$ ) decreases in the geometric mean $C_{\max }$ and AUC values of saxagliptin, respectively, with a $39 \%$ (point estimate $1.39,90 \% \mathrm{Cl} 1.23,1.56$ ) increase in the geometric mean $C_{\max }$ and no change (point estimate $1.03,90 \% \mathrm{Cl} 0.97,1.09$ ) in the AUC of 5-hydroxy saxagliptin. Similar maximum \% inhibition and area under the $\%$ inhibition-time effect curve over $24 \mathrm{~h}$ for DPP-4 activity were observed when saxagliptin was administered alone or with rifampicin. The saxagliptin total active moieties exposure (AUC) decreased by $27 \%$ (point estimate 0.73 , $90 \% \mathrm{Cl} 0.66,0.81$ ). Saxagliptin with or without rifampicin in this study was generally well tolerated.

\section{CONCLUSIONS}

Lack of change of PD effect of saxagliptin is consistent with the observed $27 \%$ reduction in systemic exposure to the total active moieties, which is not considered clinically meaningful. Based on these findings, it is not necessary to adjust the saxagliptin dose when co-administered with rifampicin. 


\section{Introduction}

Saxagliptin is a highly potent, selective, reversible, dipeptidyl peptidase-4 (DPP-4) inhibitor [1]. The DPP-4 inhibitors are an emerging new therapeutic class designed to treat type 2 diabetes [2]. The DPP-4 enzyme actively converts the key insulinotropic hormone glucagon-like peptide-1 (GLP-1) from intact GLP-1 to an inactive form and is responsible for the short half-life of intact GLP-1 in vivo [3]. Inhibitors of DPP-4 increase concentrations of endogenous intact GLP-1 and thus potentiate its physiological actions, augmenting postprandial insulin secretion and improving the overall glycaemic profile in patients with type 2 diabetes [4]. Because DPP-4 inhibitors stimulate insulin secretion in a glucose-dependent manner, this mechanism of action is expected to present low risk of hypoglycaemia and may not lead to weight gain [1].

The clinical pharmacodynamic (PD) properties of saxagliptin $5 \mathrm{mg}$ are consistent with once daily dosing. The usual clinical dose in adults is $5 \mathrm{mg}$ administered orally once daily [5]. Saxagliptin has predictable and dose-proportional pharmacokinetics (PK), with minimal accumulation with once daily dosing [6]. Upon oral administration, saxagliptin is rapidly and extensively absorbed, and is cleared via metabolic and renal routes [5]. The major pharmacologically active metabolite of saxagliptin is 5-hydroxy saxagliptin, with an in vitro DPP-4 inhibitory potency at $37^{\circ} \mathrm{C}$ of approximately one-half that of the parent compound saxagliptin [5].

In vitro metabolism studies (individual recombinant cytochrome P450 [CYP] enzymes and human liver microsomes) indicate that saxagliptin is metabolized primarily by CYP3A4 (major) and CYP3A5 (minor) enzymes to 5-hydroxy saxagliptin [5]. In vitro incubation of 5-hydroxy saxagliptin with CYP450 isozymes showed a low turnover and no specific CYP isozyme has been identified that catalyzes metabolic elimination of 5-hydroxy saxagliptin. The elimination of 5-hydroxy saxagliptin is almost exclusively by non-metabolic routes (urinary excretion [major] and biliary excretion [minor]). Ketoconazole, a potent inhibitor of CYP3A4/5 enzymes, increased the $C_{\max }$ and $A U C_{0-\infty}$ of saxagliptin in healthy subjects by approximately $62 \%$ and $145 \%$, respectively, and decreased the $C_{\max }$ and $A \cup C_{0-\infty}$ of 5 -hydroxy saxagliptin by approximately $95 \%$ and $88 \%$, respectively [7]. Similarly, diltiazem, a moderate inhibitor of CYP3A4/5, increased the $C_{\max }$ and $A \cup C_{0-\infty}$ of saxagliptin by $63 \%$ and $109 \%$, respectively, and decreased the $C_{\max }$ and $\mathrm{AUC}_{0-\infty}$ of 5-hydroxy saxagliptin by $43 \%$ and $34 \%$, respectively [8]. These in vitro studies and clinical findings clearly establish a major role of CYP3A4/5 in the disposition of saxagliptin and further indicate that concomitant administration of saxagliptin with CYP3A4/5 inducers in humans would be expected to decrease systemic exposure to saxagliptin.

Rifampicin is a potent inducer of CYP3A4 $[9,10]$, and has been shown to induce various drug efflux transporters such as P-glycoprotein (P-gp) $[9,11]$ and multi-drug resistance protein 2 (MRP2) $[12,13]$ via the pregnane $X$ receptor-dependent mechanism [14]. Recent experimental evidence also suggests that rifampicin inhibits certain uptake transporters such as organic anion transporter polypeptide (OATP-1) and MRP2 [15-19]. The European Agency for the Evaluation of Medicinal Products and the US Food and Drug Administration (FDA) recommend rifampicin as a CYP3A4 inducer for use in clinical drug-drug interaction studies in which CYP induction may be of clinical importance [20-22].

The present study was designed to examine the effect of the co-administration of saxagliptin with an archetypal CYP inducer, rifampin (rifampicin), on the PK and PD of saxagliptin, to determine if the combination is safe and tolerable and to guide any potential dose adjustment for saxagliptin when rifampicin is co-administered. Once daily dosing for 5 days with rifampicin $600 \mathrm{mg}$, which is the highest recommended once daily dose, has been shown to achieve marked CYP induction [10, 23]. Accordingly, in this study, a daily dose of rifampicin $600 \mathrm{mg}$ was administered orally for 6 days to ensure maximum induction of CYP3A4 prior to saxagliptin administration. A $5 \mathrm{mg}$ dose of saxagliptin was used in this study, because it represents the usual once daily clinical dose of saxagliptin.

\section{Methods}

This study was conducted at the Bristol-Myers Squibb Clinical Research Center, Hamilton, New Jersey, USA.

\section{Ethics}

All study participants gave written informed consent before entering the study. The study protocol and informed consent form were approved by an institutional review board (New England Institutional Review Board, Wellesley, Massachusetts, USA). The study was conducted according to federal regulations $[24,25]$ and the Declaration of Helsinki. Per statistical considerations, 14 subjects who met inclusion/exclusion criteria were needed.

\section{Subjects}

Male and female subjects 18 to 45 years of age, determined as healthy by medical history, physical examination, 12-lead electrocardiogram (ECG), routine haematology and serum chemistry analysis and urinalysis participated in the study. Female subjects were not breast-feeding, pregnant or of childbearing potential and had negative pregnancy tests within $24 \mathrm{~h}$ prior to dosing. Subjects with known allergies to DPP-4 inhibitors or rifampicin were excluded. Potential confounding effects of the use of any other medicinal agents were mitigated by excluding subjects who were on: (i) any prescription drugs or over the counter gastric acid controllers or St John's wort (CYP3A and P-gp inducer) within 4 weeks prior, (ii) any other drugs 
including over the counter medications and herbal preparations within 1 week and (iii) any injectable or implantable hormonal contraceptive agent within 3 months prior to study drug administration.

\section{Design and procedure}

This was a non-randomized, open label, single sequence study (Figure 1), in which subjects who met study requirements within 21 days prior to study enrolment were admitted to the clinical facility on day -1 . Each subject received a single oral dose of saxagliptin $5 \mathrm{mg}$ on day $1,600 \mathrm{mg}$ once daily oral doses of rifampicin on days $2-6$, and a single dose of saxagliptin $5 \mathrm{mg}$ concomitantly with a single dose of rifampicin $600 \mathrm{mg}$ on day 7. At the time of dosing, $240 \mathrm{ml}$ of water was taken by the subject orally along with study medication. Except during the rifampicin alone treatment period (days 2-6), subjects had nothing to eat or drink except water for $10 \mathrm{~h}$ prior to and until $4 \mathrm{~h}$ after study drug administration. Also, subjects did not drink water in the period $1 \mathrm{~h}$ before or after study drug administration except when dosing. Subjects remained confined to the research unit until day 8 (after the PK/PD collection and all safety assessments were completed) and were not allowed strenuous exercise, smoking or consumption of alcoholic beverages, grapefruit containing products, Seville oranges or caffeine containing food or beverages during the study.

\section{Safety assessments}

Physical examinations, vital sign measurements, 12-lead ECGs and clinical laboratory evaluations (haematology studies, serum chemistry analysis and urinalysis) were performed at selected times throughout the study. Subjects were closely monitored for any adverse events (AEs) throughout the study.

\section{PK and PD sampling}

Blood samples were collected for PK (plasma saxagliptin and 5-hydroxy saxagliptin concentrations) and PD (plasma
DPP-4 activity) prior to saxagliptin dosing and at $0.25,0.5$, $1,1.5,2,3,5,8,12,16$ and $24 \mathrm{~h}$ after saxagliptin dosing on days 1 and 7. Urine samples were collected prior to dosing and during the following intervals: $0-12 \mathrm{~h}$ and $12-24 \mathrm{~h}$ after saxagliptin dosing on days 1 and 7. The first collection interval (0-12 h) began with a complete void at pre-dose and each interval concluded with a complete void. The volume of urine obtained was recorded at the end of each collection interval. The plasma (PK and PD) and urine samples were stored at $-20^{\circ} \mathrm{C}$ until analysis within the known period of stability.

\section{Quantitative determination of saxagliptin and} 5-hydroxy saxagliptin in plasma and urine

Saxagliptin and its major active metabolite, 5-hydroxy saxagliptin, were analyzed in the plasma and urine samples using a validated liquid chromatography-tandem mass spectrometry (LC-MS/MS) method. Sample preparation for plasma and urine samples involved solid phase extraction, and saxagliptin- ${ }^{13} \mathrm{CD}_{2}$ and 5 -hydroxy saxagliptin- ${ }^{13} \mathrm{CD}_{2}$ were used as internal standards. The lower limits of quantitation (LLQ) of the assays were $1 \mathrm{ng} \mathrm{m}^{-1}$ for saxagliptin and $2 \mathrm{ng} \mathrm{ml}^{-1}$ for 5-hydroxy saxagliptin in plasma, and $5 \mathrm{ng} \mathrm{ml}^{-1}$ for saxagliptin and $10 \mathrm{ng} \mathrm{ml}^{-1}$ for 5 -hydroxy saxagliptin in urine. The accuracy and precision values for the assay were well within the widely accepted criteria of $\pm 15 \%$ deviation and coefficient of variation (CV) percentage set forth for quantitative determination of an analyte in biological matrices [26]. All samples for a subject from both treatments (with and without rifampicin) were analyzed together. Stability of both saxagliptin and 5-hydroxy saxagliptin in human plasma was established for 12 months at $-20^{\circ} \mathrm{C}$ and for 736 days in human urine at $-20^{\circ} \mathrm{C}$, and all samples were analyzed within this period of analyte stability.

\section{Plasma DPP-4 (PD) activity determination}

Plasma samples were analyzed to determine DPP-4 activity using a validated endpoint enzyme activity assay. Briefly,

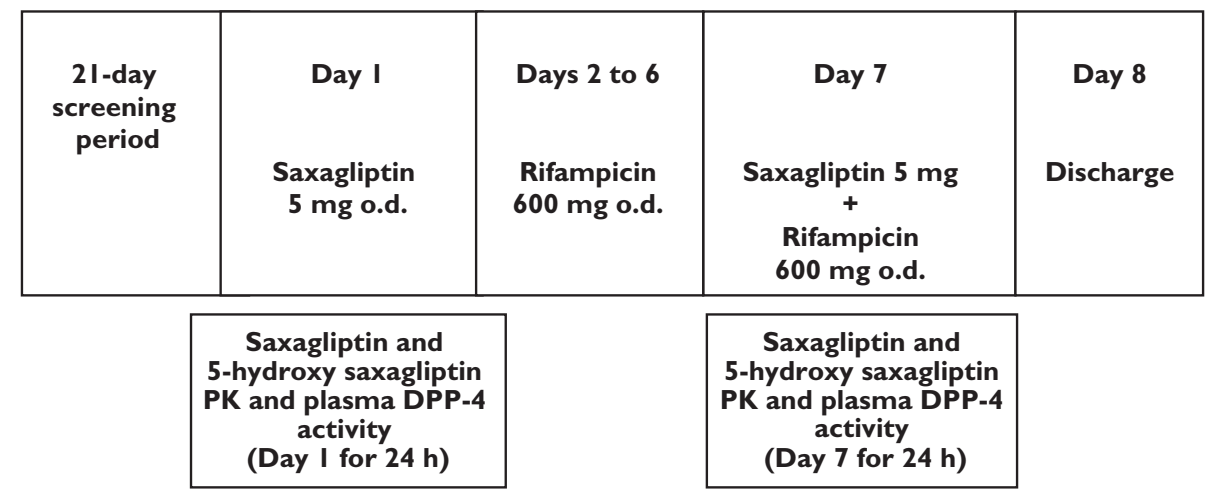

Figure 1

A schematic of the study design 
$10 \mu \mathrm{l}$ of plasma was incubated with $100 \mu \mathrm{l}$ of $2000 \mu \mathrm{mol} \mathrm{I}^{-1}$ glycyl-prolyl-p-nitro-aniline for $90 \mathrm{~min}$ at $\mathrm{pH} 7.4$ and $25^{\circ} \mathrm{C}$ and the rate of $\mathrm{p}$-nitro-aniline product formation was determined as a measure of DPP-4 activity. The measured change in absorbance at $405 \mathrm{~nm}$ (p-nitro-aniline formation) was directly proportional to the plasma DPP-4 activity. The reportable assay range was $0.31-40 \mathrm{UI}^{-1}$. Deviations of the mean measured quality control samples were within $\pm 1.7 \%$ and the between-run and within-run variability was $\leq 6.55 \% \mathrm{CV}$.

\section{PK analysis}

PK parameters were generated by non-compartmental methods and other specific methods, as described following using the program Kinetica (version 4.4.1, Thermo Electron Corporation, Philadelphia, Pennsylvania, USA). The metabolite : parent $A U C_{0-\infty}$ ratio was calculated by dividing the molar $\mathrm{AUC}_{0-\infty}$ value for the metabolite, 5-hydroxy saxagliptin, by the molar $A \cup C_{0-\infty}$ value for the parent drug, saxagliptin. The saxagliptin total active moiety exposure values were calculated by adding the molar $A \cup C_{0-\infty}$ value for saxagliptin to one-half of the molar $A \cup C_{0-\infty}$ value for 5-hydroxy saxagliptin. One-half of the 5-hydroxy saxagliptin $A_{U} C_{0-\infty}$ value was used to account for the 2 -fold lower in vitro potency for DPP-4 inhibition of 5-hydroxy saxagliptin relative to that of saxagliptin [5].

\section{PD analysis}

PD parameters were generated by non-compartmental methods using the program Kinetica. Prior to noncompartmental analysis of the plasma DPP-4 activity-time data, DPP-4 activity-time data were converted to percentage inhibition of plasma DPP-4 activity (DPP-4 \% inhibition) using each individual subject's baseline DPP-4 activity, i.e. DPP-4 activity prior to saxagliptin dosing for each treatment period (pre-dose, time $0 \mathrm{~h}$ ). The following formula was used:

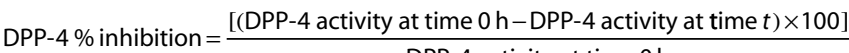
DPP-4 activity at time $0 \mathrm{~h}$

\section{Statistical analysis}

The log-transformed PK parameters $\left(C_{\max }, \mathrm{AUC}_{0-\infty}\right)$ for saxagliptin and 5-hydroxy saxagliptin were analyzed using analysis of variance (ANOVA). Point estimates and $90 \%$ confidence intervals for treatment differences on the log scale were exponentiated to obtain point estimates and $90 \%$ confidence intervals for the day 7 (saxagliptin with rifampicin) and day 1 (saxagliptin alone) ratio of geometric means for $C_{\max }$ and $A \cup C_{0-\infty}$ of saxagliptin and 5-hydroxy saxagliptin, on the original scale of measurement. Secondary PK and PD parameters and safety variables were analyzed in a descriptive manner by summary statistics. All statistical analyses were performed using SAS software (SAS Institute, Inc., Cary, North Carolina, USA).

\section{Analysis of pooled urine samples to evaluate relative changes in metabolites}

Two pooled urine samples from the two treatments representing day 7 (saxagliptin with rifampicin) and day 1 (saxagliptin alone) were prepared by combining equal volumes (100 $\mu \mathrm{l}$ each) from both the $0-12 \mathrm{~h}$ and $12-24 \mathrm{~h}$ post-dose cumulative urine samples of all the subjects for the two treatments separately. The pooled human urine samples were centrifuged at $13000 \mathrm{rpm}$ for $5 \mathrm{~min}$ in a microcentrifuge and $50 \mu \mathrm{l}$ portions were injected and analyzed by positive ion electrospray LC-MS/MS. Metabolites of saxagliptin that had been previously identified in human studies were monitored using selected multiple reaction monitoring transitions. The peak areas of these metabolites in the two samples were determined and relative changes in metabolite amounts were reported as the peak area ratio.

\section{Results}

Fourteen subjects were enrolled and received study drug. The baseline demographics and characteristics of the subjects are given in Table 1. One subject withdrew consent prior to dosing on day 5 . This subject was included in safety assessments because he had been dosed with study medications, but he was excluded from PK and PD evaluation data sets due to incomplete data.

\section{Safety and tolerability}

No deaths, serious AEs or discontinuations due to AEs were reported during the study. Eight AEs occurred in six subjects. All the AEs were determined to be of mild intensity by the investigator. The most common AE was headache, which occurred in one subject (7.1\%) following administration of saxagliptin $5 \mathrm{mg}$ alone, one subject (7.1\%) following administration of rifampicin $600 \mathrm{mg}$ once daily alone and three subjects (23.1\%) following co-administration of saxagliptin $5 \mathrm{mg}$ and rifampicin $600 \mathrm{mg}$. One subject reported

\section{Table 1}

Baseline demographics and characteristics

\begin{tabular}{|lc|}
\hline & All subjects $\boldsymbol{~}$ \\
\hline Age (years) Mean (SD) & $33(7)$ \\
Gender, $\boldsymbol{n}(\%)$ & \\
Male & $13(93)$ \\
Female & $1(7)$ \\
Race, $\boldsymbol{n}(\%)$ & \\
White & $2(14)$ \\
Black/African American & $11(79)$ \\
Other & $1(7)$ \\
Weight (kg) Mean (SD) & $81.7(10.0)$ \\
BMI (kg m $\mathbf{~}^{-2}$ ) Mean (SD) & $26.3(2.7)$ \\
\hline
\end{tabular}

$† N=14 . \mathrm{BMI}$, body mass index; SD, standard deviation. 
A Saxagliptin

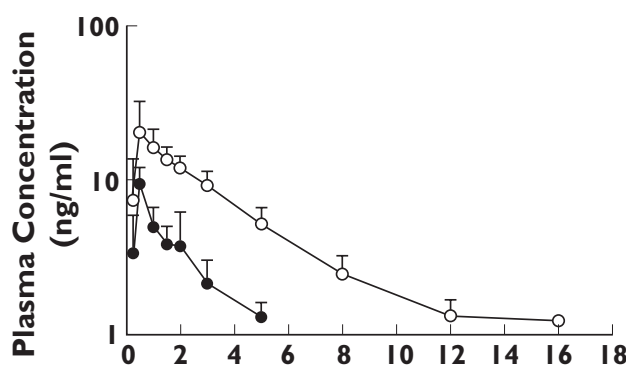

B 5-Hydroxy saxagliptin

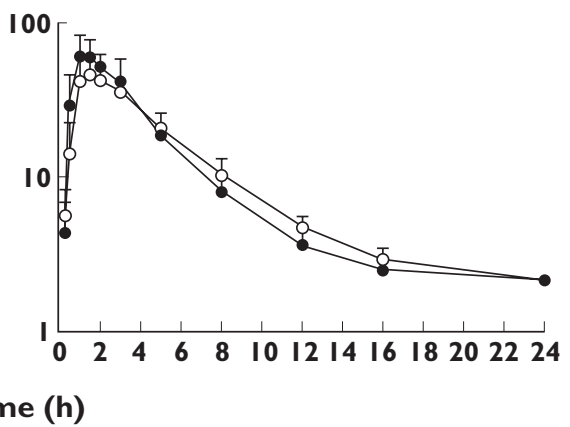

\section{Figure 2}

Mean (+SD, $n=13$ ) plasma concentration-time profiles for (A) saxagliptin and (B) 5-hydroxy saxagliptin (major active metabolite of saxagliptin) following administration of a single oral dose of saxagliptin $5 \mathrm{mg}$ to healthy subjects with and without rifampicin (600 mg once daily for 6 days). Saxagliptin $5 \mathrm{mg}$ $(\multimap-$ ); Saxagliptin $5 \mathrm{mg}$ + rifampicin $600 \mathrm{mg}(\longrightarrow)$

\section{Table 2}

Summary of saxagliptin pharmacokinetic parameters following administration of a single oral dose of saxagliptin 5 mg to healthy subjects with and without rifampicin ( $600 \mathrm{mg}$ once daily for 6 days)

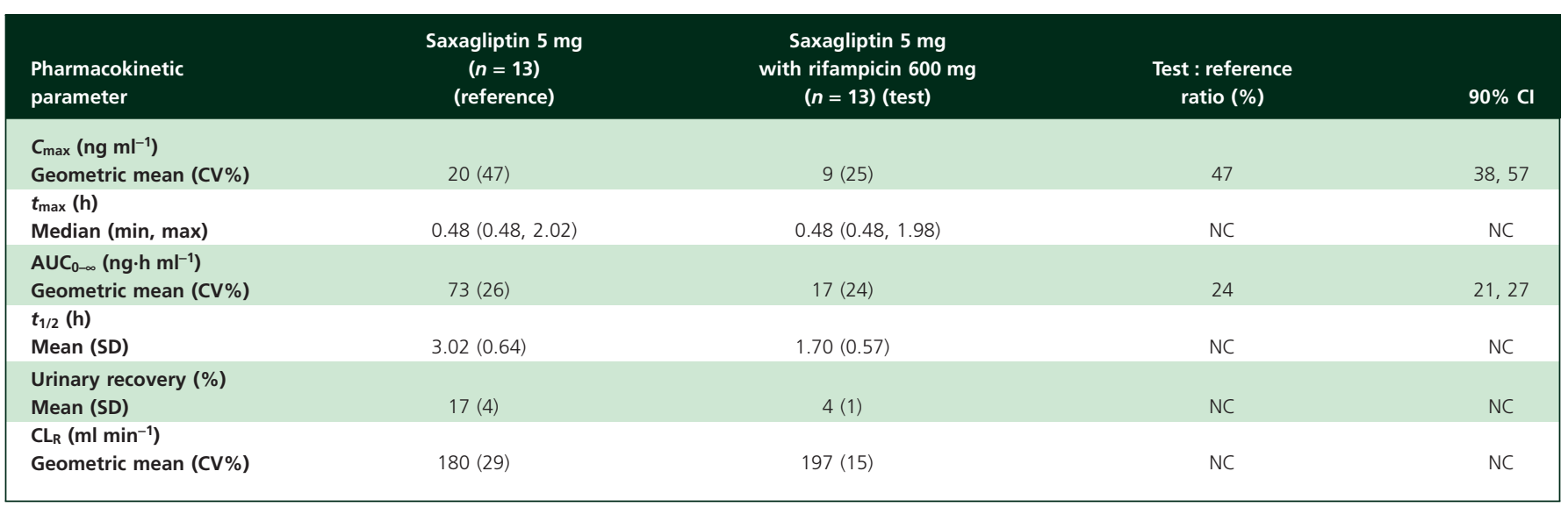

$\mathrm{AUC}_{0-\infty}$, area under the plasma concentration-time curve from time 0 to infinity; $\mathrm{Cl}$, confidence interval; $\mathrm{CL}_{\mathrm{R}}$, apparent renal clearance; $\mathrm{C}_{\text {max }}$ maximum plasma concentration; $\mathrm{CV}_{\mathrm{O}}$, coefficient of variation $\%$; $N C$, not calculated; $t_{1 / 2}$, terminal elimination half-life; $t_{\max }$, time to reach the $C_{\max }$.

abdominal pain and one other subject reported pain and swelling at the venipuncture site following administration of rifampicin $600 \mathrm{mg}$. No clinically significant changes or trends were observed for ECGs, vital signs, clinical laboratory tests or physical examinations.

\section{Effect of rifampicin on the PK of saxagliptin}

The mean plasma concentration-time profiles of saxagliptin and 5-hydroxy saxagliptin are shown in Figure 2. PK parameter estimates for saxagliptin and 5-hydroxy saxagliptin are summarized in Table 2 and Table 3, respectively.

Co-administration of rifampicin with saxagliptin substantially reduced saxagliptin exposure. When saxagliptin $5 \mathrm{mg}$ was co-administered with rifampicin $600 \mathrm{mg}$, the geometric means for $C_{\max }$ and $\mathrm{AUC}_{0-\infty}$ of saxagliptin decreased by $53 \%$ (point estimate $0.47,90 \% \mathrm{Cl} 0.38,0.57$ ) and $76 \%$ (point estimate $0.24,90 \% \mathrm{Cl} 0.21,0.27$ ), respectively, relative to those observed following administration of saxagliptin $5 \mathrm{mg}$ alone. The $90 \% \mathrm{Cls}$ for the ratios of population geometric means, with and without rifampicin, were entirely below the pre-specified no-effect interval of $80 \%$ to $125 \%$ recommended by the US $[20,21]$ and the European Union [22] regulatory guidances for the conduct of in vivo drug interaction studies for $C_{\max }$ and $A \cup C_{0-\infty}$. Consistent with the decrease in saxagliptin exposure was a reduction in the $t_{1 / 2}$ of saxagliptin from $3.02 \mathrm{~h}$ to $1.70 \mathrm{~h}$ upon co-administration of saxagliptin with rifampicin. The $t_{\max }$ of saxagliptin was unaffected by rifampicin, and no substantial changes in the renal clearance values of saxagliptin were observed when saxagliptin was administered with or without rifampicin treatment. 


\section{Table 3}

Summary of 5-hydroxy saxagliptin (major active metabolite of saxagliptin) pharmacokinetic parameters following administration of a single oral dose of saxagliptin $5 \mathrm{mg}$ to healthy subjects with and without rifampicin ( $600 \mathrm{mg}$ once daily for 6 days)

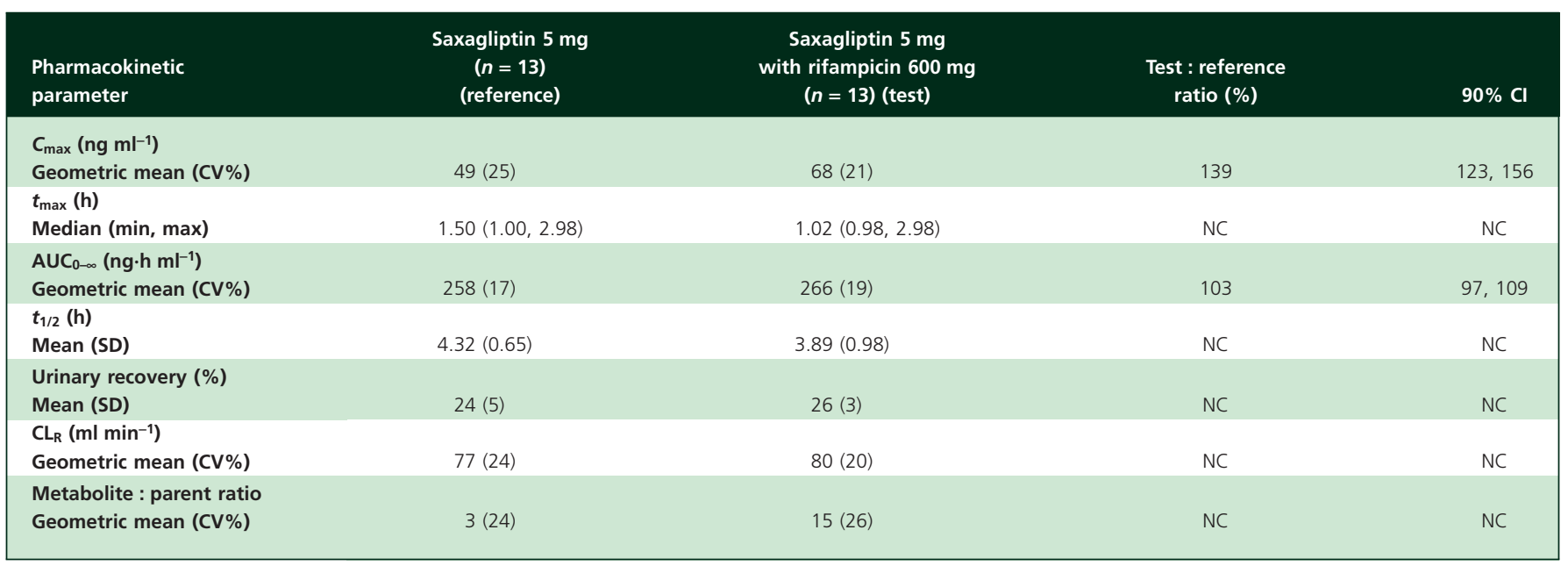

$\mathrm{AUC}_{0-\infty}$, area under the plasma concentration-time curve from time 0 to infinity; $\mathrm{Cl}$, confidence interval; $\mathrm{CL}_{\mathrm{R}}$, apparent renal clearance; $\mathrm{C}_{\mathrm{max}}, \mathrm{maximum}$ plasma concentration; $\mathrm{CV} \%$, coefficient of variation \%; NC, not calculated; metabolite : parent ratio, (5-hydroxy saxagliptin $\mathrm{AUC}_{0-\infty}$ : $\left.\operatorname{saxagliptin} A \cup \mathrm{C}_{0-\infty}\right) \times(315.42 / 331.42)$; $t_{1 / 2}$, terminal elimination half-life; $t_{\max }$, time to reach the $C_{\max }$.

In contrast to the parent compound saxagliptin, the PK profile of 5-hydroxy saxagliptin was not dramatically affected by rifampicin co-administration. When saxagliptin $5 \mathrm{mg}$ was co-administered with rifampicin $600 \mathrm{mg}$, the geometric means for $C_{\max }$ increased by $39 \%$ (point estimate $1.39,90 \% \mathrm{Cl} 1.23,1.56)$ without change in the $\mathrm{AUC}_{0-\infty}$ (point estimate $1.03,90 \% \mathrm{Cl} 0.97,1.09$ ) of 5-hydroxy saxagliptin, relative to those observed following administration of saxagliptin $5 \mathrm{mg}$ alone. The $90 \% \mathrm{Cls}$ for the ratios of population geometric means, with and without rifampicin, were within the $80 \%$ to $125 \%$ no-effect interval for $A U C_{0-\infty}$ of 5-hydroxy saxagliptin. However, the $90 \% \mathrm{Cl}$ for the ratio of population geometric means, with and without rifampicin, extended above the no-effect interval for $C_{\max }$ of 5-hydroxy saxagliptin.

There was a 5-fold increase in the metabolite: parent ratio when saxagliptin was co-administered with rifampicin compared with saxagliptin alone. No substantial changes in renal clearance values or $t_{\max }$ and $t_{1 / 2}$ of 5-hydroxy saxagliptin were observed when saxagliptin was administered with and without rifampicin treatment. The geometric mean for saxagliptin total active moiety exposure $\left(\mathrm{AUC}_{0-\infty}\right.$ ) decreased by $27 \%$ (point estimate $0.73,90 \% \mathrm{Cl} 0.66,0.81)$ when saxagliptin was co-administered with rifampicin compared with when saxagliptin was administered alone.

\section{Effect of rifampicin on the PD profile (plasma $D P P-4$ activity) of saxagliptin}

The mean plasma DPP-4 activity-time (percentage change from baseline, where baseline equals DPP-4 activity at pre-dose) profiles for saxagliptin administered with and

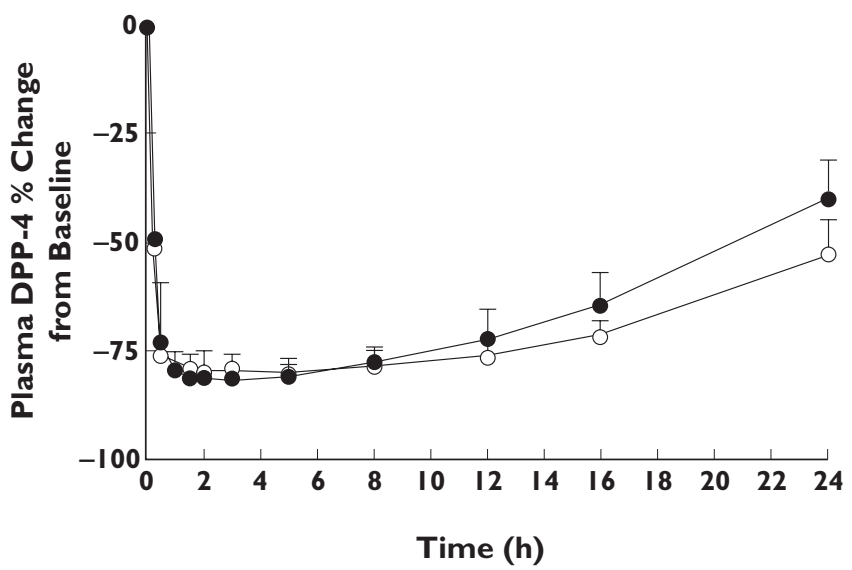

\section{Figure 3}

Mean (+SD, $n=13$ ) plasma DPP-4 activity (change from baseline expressed as a negative value of DPP- $4 \%$ inhibition) time profiles following administration of a single oral dose of saxagliptin $5 \mathrm{mg}$ to healthy subjects with and without rifampicin ( $600 \mathrm{mg}$ once daily for 6 days). Saxa-

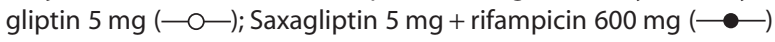

without rifampicin treatment are shown in Figure 3. PD parameter estimates for plasma DPP-4 activity are summarized in Table 4. The geometric mean maximum inhibition of DPP-4 activity $\left(\% l_{\max }\right)$ remained unchanged (point estimate $1.00,90 \% \mathrm{Cl} 0.98,1.03)$ and the overall inhibition of DPP-4 activity over the dosing interval (i.e. the $A U E C_{0-24}$ ) was also unchanged (point estimate $0.94,90 \% \mathrm{Cl} 0.90,0.97$ ) when saxagliptin was administered with rifampicin relative to that observed following administration of saxagliptin $5 \mathrm{mg}$ alone. 


\section{Table 4}

Summary of plasma DPP-4 \% inhibition pharmacodynamic parameters following administration of a single oral dose of saxagliptin 5 mg to healthy subjects with and without rifampicin (600 mg once daily for 6 days)

\begin{tabular}{|c|c|c|c|}
\hline Pharmacodynamic parameter & $\begin{array}{c}\text { Saxagliptin } 5 \mathrm{mg} \\
\quad(n=13) \\
\text { (reference) }\end{array}$ & $\begin{array}{l}\text { Saxagliptin } 5 \mathrm{mg} \text { with } \\
\text { rifampicin } 600 \mathrm{mg} \\
\text { ( }(n=13) \text { (test) }\end{array}$ & $\begin{array}{l}\text { Point estimate }(90 \% \mathrm{Cl}) \\
\text { Ratio of geometric means } \\
\text { test : reference }\end{array}$ \\
\hline$\% I_{\max }$ Mean (SD) & $83.07(3.50)$ & $83.22(2.14)$ & $1.00(0.98,1.03)$ \\
\hline$t_{\max }($ effect) $(\mathrm{h})$ Median (min, max) & $2.00(1.00,8.00)$ & $2.00(1.00,12.00)$ & \\
\hline AUEC $_{0-24}(\%$ inhibited.h) Mean (SD) & $1711.61(71.82)$ & $1603.97(111.20)$ & $0.94(0.90,0.97)$ \\
\hline$t_{1 / 2}($ effect) $(h)$ Mean (SD) & $25.85(10.64)$ & $14.46(4.23)$ & \\
\hline
\end{tabular}

DPP-4 $\%$ inhibition $=\frac{[(\text { DPP }-4 \text { activity at time } 0 \mathrm{~h}-\text { DPP }-4 \text { activity at time } t) \times 100]}{\text { DPP- } 4 \text { activity at time } 0 \mathrm{~h}}$

AUEC 0-24, area under the DPP-4 \% inhibition (effect)-time curve from time 0 to the last measured time point 24 h post-dose; Cl, confidence interval; \% /max, peak DPP-4 \% inhibition; $t_{\max }$ (effect), time to reach peak DPP-4 \% inhibition; $t_{1 / 2}$ (effect), apparent effect terminal half-life.

\section{Table 5}

Peak area ratios for saxagliptin metabolites from the pooled urine samples following administration of a single oral dose of saxagliptin 5 mg to healthy subjects with and without rifampicin (600 mg once daily for 6 days)

\begin{tabular}{|c|c|c|c|c|c|}
\hline Analyte in urine & $\begin{array}{l}\text { Structural description of } \\
\text { analyte in urine }\end{array}$ & $\begin{array}{l}\text { Peak a } \\
\text { Saxagliptin } 5 \text { mg } \\
\text { (reference) }\end{array}$ & $\begin{array}{l}\text { ounts) } \\
\text { Saxagliptin } 5 \mathrm{mg} \\
\text { with rifampicin } \\
600 \mathrm{mg} \text { (test) }\end{array}$ & $\begin{array}{l}\text { Peak area ratio } \\
\text { Test : } \\
\text { reference }\end{array}$ & $\begin{array}{l}\text { Transition } \\
\text { Multiple reaction } \\
\text { monitoring }\end{array}$ \\
\hline Saxagliptin & Parent & 5390000 & 1290000 & 0.24 & $316.0 / 180.0$ \\
\hline D1 & Degradant & 8080 & 2080 & 0.26 & $316.0 / 288.0$ \\
\hline M1 & Mono-hydroxy & 64300 & 50500 & 0.79 & $332.0 / 196.0$ \\
\hline M2 (5-hydroxy saxagliptin) & Mono-hydroxy (major metabolite) & 3820000 & 3460000 & 0.91 & $332.0 / 196.0$ \\
\hline M3 & Mono-hydroxy & 394000 & 206000 & 0.52 & $332.0 / 196.0$ \\
\hline M5 & Di-hydroxy & 31100 & 130000 & 4.18 & $348.0 / 212.0$ \\
\hline M8 & Di-hydroxy & 34700 & 73300 & 2.11 & $348.0 / 212.0$ \\
\hline M13 & Mono-hydroxy & 3460 & 4030 & 1.16 & $332.0 / 304.0$ \\
\hline M16 & Mono-hydroxy & 31600 & 35000 & 1.11 & $332.0 / 196.0$ \\
\hline M17 & Di-hydroxy & 15700 & 40400 & 2.57 & $348.0 / 212.0$ \\
\hline M19 & Gluc-mono-hydroxy & 2870 & 3220 & 1.12 & $508.0 / 332.0$ \\
\hline M27 & Mono-hydroxy-decyanated & 14200 & 55300 & 3.89 & $321.0 / 196.0$ \\
\hline M40 & Gluc-mono-hydroxy & 1340 & 1360 & 1.01 & $508.0 / 332.0$ \\
\hline M45 & Sulfate-parent & 2930 & 673 & 0.23 & $396.0 / 298.0$ \\
\hline M46 & Gluc-parent & 14300 & 3080 & 0.22 & $492.0 / 316.0$ \\
\hline M49 & Di-hydroxy & 10400 & 32600 & 3.13 & $348.0 / 212.0$ \\
\hline
\end{tabular}

\section{Relative changes in the amount of metabolites in the pooled urine samples}

Peak areas for parent saxagliptin and each metabolite in the two pooled urine samples from saxagliptin with and without rifampicin are summarized in Table 5. Also summarized in Table 5 is the peak area ratio for each metabolite in the two treatments. Peak area of each metabolite during saxagliptin and rifampicin co-administration treatment was divided by peak area of that metabolite during treatment with saxagliptin alone. With rifampicin there was a small (2- to 5.5-fold) increase in formation of several minor dihydroxy metabolites and a glucuronide conjugate of saxagliptin. However, the concentrations of these metabolites in the urine samples were minor compared with saxagliptin or 5-hydroxy saxagliptin.

\section{Discussion}

\section{Safety and tolerability}

The types of AEs and their frequency reported by healthy subjects were not unusual for short-term studies in healthy subjects, and did not show a clear difference for the two treatments (saxagliptin administered with and without rifampicin). This result suggests that a single dose of saxagliptin $5 \mathrm{mg}$ was generally safe and well tolerated when co-administered with rifampicin.

\section{Pharmacokinetics}

Co-administration of rifampicin with saxagliptin decreased the systemic exposure of saxagliptin (53\% and $76 \%$, respective decrease in $C_{\max }$ and $\mathrm{AUC}_{0-\infty}$ values) and 
increased the $C_{\max }$ of its pharmacologically active major metabolite, 5 -hydroxy saxagliptin, by $39 \%$, consistent with an increase in CYP3A-mediated metabolism. However, co-administration of rifampicin with saxagliptin did not change the overall systemic exposure $\left(A \cup C_{0-\infty}\right)$ to 5 -hydroxy saxagliptin. While the percentage of saxagliptin dose recovered in urine was lower when rifampicin was administered with saxagliptin, there were no changes in the renal clearance of either saxagliptin or 5-hydroxy saxagliptin when saxagliptin was administered with or without rifampicin. The total active moieties (molar summations of saxagliptin exposure parameter with one-half the molar exposure parameter for 5-hydroxy saxagliptin, factoring in the 2-fold higher inhibition constant $\left(K_{\mathrm{i}}\right)$ value of 5-hydroxy saxagliptin towards in vitro DPP-4 inhibition at $37^{\circ} \mathrm{C}[5$, Bristol-Myers Squibb/AstraZeneca, unpublished data]) $\mathrm{AUC}_{0-\infty}$ was reduced by $27 \%$ on co-administration of saxagliptin with rifampicin. In line with the PK findings for the total active moieties, the maximum inhibition of DPP-4 activity $\left(\% I_{\max }\right)$ and the overall inhibition of DPP-4 activity over the dosing interval, as measured by $\mathrm{AUEC}_{0-24}$, remained unchanged when saxagliptin was administered with rifampicin, compared with when it was administered alone.

The decrease in systemic exposure to saxagliptin when saxagliptin is co-administered with rifampicin can be attributed mainly to induction of CYP3A4/5-mediated metabolism of saxagliptin by rifampicin. This conclusion is supported by the observation of a higher $C_{\max }$ of 5-hydroxy saxagliptin, indicating induced first-pass metabolism when saxagliptin is co-administered with rifampicin. Metabolic induction is also evidenced by the 5-fold increase in metabolite:parent $A U C_{0-\infty}$ ratio. Metabolic induction of saxagliptin also resulted in a decrease in the $t_{1 / 2}$ of saxagliptin from $3.02 \mathrm{~h}$ when saxagliptin was administered alone to $1.70 \mathrm{~h}$ when saxagliptin was co-administered with rifampicin.

When co-administered with saxagliptin, rifampicin did not alter renal clearance of either saxagliptin or 5-hydroxy saxagliptin, indicating that rifampicin did not induce renal transporters that may be involved in active renal secretion of saxagliptin. However, the amount of total administered dose recovered in urine (i.e. percentage urinary recovery of saxagliptin +5-hydroxy saxagliptin) was lower when rifampicin was co-administered with saxagliptin (30\%) compared with when saxagliptin was administered alone $(41 \%)$. Thus, a fraction of the saxagliptin dose when co-administered with rifampicin is not accounted for in urine. Renal clearance of saxagliptin and 5-hydroxy saxagliptin is not altered by rifampicin; the implication of this finding is that a fraction of the saxagliptin dose in the systemic circulation (as measured by saxagliptin and 5 -hydroxy saxagliptin PK) is also not accounted for. This is likely the source of the lower than expected impact of rifampicin on 5-hydroxy saxagliptin $\mathrm{AUC}_{0-\infty}$.

A number of possible explanations exist for these findings. If the fraction of saxagliptin metabolized to 5-hydroxy saxagliptin was increased by rifampicin, the less than expected increase in the systemic AUC of 5-hydroxy saxagliptin may have been due to increased clearance of 5-hydroxy saxagliptin by non-renal pathways. Hence, the observation of decreased systemic exposure of saxagliptin without a subsequent larger increase in the AUC of 5-hydroxy saxagliptin than was observed could be explained by the hypothesis that rifampicin may have induced non-renal elimination pathways of 5-hydroxy saxagliptin, such as biliary/intestinal secretion of 5-hydroxy saxagliptin [Bristol-Myers Squibb/AstraZeneca, unpublished data]. In vivo animal experiments indicate that oral bioavailability of 5-hydroxy saxagliptin is very poor, suggesting that there is minimal likelihood of substantial intestinal resorption of 5-hydroxy saxagliptin secreted via bile or intestinal secretion. The increased biliary/intestinal secretion hypothesis in the rifampicin-induced state is consistent with the observation of a $39 \%$ increase in the $C_{\max }$ of 5-hydroxy saxagliptin (larger fraction metabolized by first pass), but no increase in its AUC (increased nonrenal clearance).

When saxagliptin is administered alone, 5-hydroxy saxagliptin undergoes minimal further metabolism. The possibility that rifampicin may have induced alternate metabolic pathways, potentially catalyzing further metabolism of 5-hydroxy saxagliptin or catalyzing conversion of saxagliptin to metabolites other than 5-hydroxy saxagliptin, was tested using leftover urine samples from both treatment periods to identify whether the extent of formation of previously known minor metabolites had increased. Urine samples from both treatments were analyzed by LC-MS/MS and peak area ratios of each metabolite were determined (peak area of each metabolite during saxagliptin and rifampicin co-administration treatment was divided by peak area of that metabolite during saxagliptin alone treatment). Results of this analysis indicated that there was a small (2- to 5.5-fold) increase in formation of several minor dihydroxy metabolites and a glucuronide conjugate of saxagliptin. However, due to the comparatively much lower concentrations of these metabolites $v$. saxagliptin or 5-hydroxy saxagliptin in the urine samples, the overall small magnitude increase in peak areas of these minor metabolites was not considered sufficient evidence for induction of alternate metabolic pathways of saxagliptin or 5-hydroxy saxagliptin.

Apart from its induction effect, there is experimental evidence to suggest that rifampicin also inhibits certain uptake transporters [15-19]. Clinically relevant drug-drug interaction studies have implicated rifampicin in inhibiting the OATP-1-mediated liver uptake of substrate drugs such as bosentan [16], atorvastatin [17] and atrasentan [15]. Similarly, rifampicin has also been shown to mediate drug-drug interactions via inhibition of MRP2 transport [19]. Even though saxagliptin has a low in vitro permeability, oral absorption is high, indicating that one or more as yet unidentified uptake transporters may facilitate oral absorption of saxagliptin. Rifampicin, however, may reduce 
the oral bioavailability of saxagliptin by inhibiting these putative uptake transporter(s). This hypothesis is also supported by the previously discussed reduction in the amount of total dose recovered in urine (percentage urinary recovery of saxagliptin +5-hydroxy saxagliptin) when saxagliptin was co-administered with rifampicin.

The decrease in systemic exposure to saxagliptin when saxagliptin is co-administered with rifampicin might also be explained, in part, by induction of P-gp-mediated efflux of saxagliptin in the intestine by rifampicin. This effect may be one reason for not observing subsequent larger increases in systemic exposures to 5-hydroxy saxagliptin. However, in vitro experiments indicate that saxagliptin is only a weak substrate of P-gp, and although induction of P-gp-mediated decreases in systemic availability of saxagliptin by rifampicin cannot be ruled out, this appears to be an unlikely mechanism for these observations [Bristol-Myers Squibb/AstraZeneca, unpublished data].

In summary, metabolic clearance of saxagliptin was increased by co-administration with rifampicin and the metabolic product was primarily 5-hydroxy saxagliptin. Systemic exposure to 5-hydroxy saxagliptin, as measured by AUC, was practically unchanged by rifampicin co-administration, whereas an increase in 5-hydroxy saxagliptin AUC was anticipated. Approximately $11 \%$ less of the saxagliptin dose was recovered in urine when saxagliptin was administered with rifampicin compared with when saxagliptin was administered alone, and there were no changes in renal clearance of the two analytes. The effects of rifampicin on 5-hydroxy saxagliptin AUC may be due to an increase in the excretion or clearance of 5-hydroxy saxagliptin or a decreased absorption of saxagliptin from the gastrointestinal tract. These findings for 5-hydroxy saxagliptin may be due to the impact of rifampicin on transporters, suggesting that the results of this study may not be generalizable to all CYP3A inducers, which may or may not affect the same transporters as rifampicin.

\section{Pharmacodynamics}

Inhibition of DPP-4 activity is the mechanism of action of saxagliptin. The DPP-4 enzyme actively converts the key insulinotropic hormone GLP-1 from intact GLP-1 to an inactive form and is responsible for the short half-life of intact GLP-1 in vivo. Inhibition of DPP-4 has been shown to increase GLP-1 exposure, resulting in increased postprandial insulin secretion and an improved glycaemic profile in patients with type 2 diabetes. Thus, inhibition of DPP-4 is the first step in the antidiabetic activity of saxagliptin, making it a reliable biomarker for the clinical effect of saxagliptin. Saxagliptin, via inhibition of DPP-4, increases concentrations of endogenous intact GLP-1, and thus potentiates its physiological actions, augmenting postprandial insulin secretion and improving the overall glycaemic profile in patients with type 2 diabetes.

Despite the higher metabolite : parent ratio resulting from rifampicin co-administration over a $24 \mathrm{~h}$ period, there was no meaningful impact on plasma DPP-4 inhibitory activity following a $5 \mathrm{mg}$ dose of saxagliptin with rifampicin compared with the same dose of saxagliptin administered alone. This finding was consistent with the difference in saxagliptin's total active moiety exposure, suggesting that the total active moieties may be a reasonable surrogate from which to estimate overall DPP-4 inhibition when metabolite:parent ratios change due to disease or co-administered drugs.

Based on these results, it appears that 5-hydroxy saxagliptin plays an important role in plasma DPP-4 inhibition in humans. Presumably, the relative changes in the 5-hydroxy saxagliptin:saxagliptin ratio observed in plasma following co-administration of saxagliptin with rifampicin are paralleled in the tissues (i.e. gut and liver), where DPP-4 inhibition is thought to be important in terms of efficacy. Overall, on the basis of plasma DPP-4 activity, a dose adjustment for saxagliptin when co-administered with rifampicin appears unnecessary.

In conclusion, co-administration of saxagliptin with the strong CYP3A inducer rifampicin was generally safe and well tolerated. During rifampicin co-administration, as expected with induction of CYP3A4/5, there was a decrease in systemic exposure to saxagliptin $\left(C_{\max }\right.$ and $A \cup C_{0-\infty}$ ), with a corresponding increase in $C_{\max }$ of the major pharmacologically active metabolite, 5-hydroxy saxagliptin. Overall systemic exposures to the total active moieties showed slight reductions, and there was no change in the maximum DPP-4 inhibition or $\mathrm{AUEC}_{0-24}$ for saxagliptin when saxagliptin was co-administered with rifampicin. This lack of change in PD effect (plasma DPP-4 activity) is consistent with the observed $27 \%$ reduction in systemic exposure to the total active moieties of saxagliptin following a $5 \mathrm{mg}$ dose, and is thus not considered clinically meaningful. Based on these findings, it is not necessary to adjust the $5 \mathrm{mg}$ saxagliptin dose when it is co-prescribed with rifampicin. Since the PK of saxagliptin are linear over a wide dose range $(2.5-400 \mathrm{mg})$, the lack of dose adjustment for saxagliptin when co-administered with rifampicin also applies to the $2.5 \mathrm{mg}$ saxagliptin dose. The observation of lack of substantial change in saxagliptin PD and the recommendation for no saxagliptin dosage adjustment with rifampicin co-administration made from data gathered in healthy subjects in this study are directly applicable to the target patient population, since the PK and PD (plasma DPP-4 activity) characteristics of saxagliptin are similar in healthy subjects compared with subjects with type 2 diabetes.

\section{Competing Interests}

This study was funded by Bristol-Myers Squibb Company and AstraZeneca. All authors are employees and shareholders of Bristol-Myers Squibb Company. 


\section{Statement of approval}

All of the authors have participated in developing this manuscript and approve of the final draft for submission.

The authors would like to acknowledge the analytical and technical support provided by Tandem Labs (West Trenton, NJ) and ICON Laboratory (Farmingdale, NY) as well as in-house support provided by Xiaohui $\mathrm{Xu}, \mathrm{PhD}$, and Fizal Nabbie for oversight of the bioanalytical aspects and the DPP-4 enzyme assay, respectively. We thank investigator John Coumbis, MD, and the research staff at the Bristol-Myers Squibb Clinical Research Center (Hamilton, NJ) for the conduct of the study, Yi-Hao Yu, MD, PhD, for his medical opinion in the development of the study protocol and Ramaswamy lyer, PhD, and Lisa Christopher, PhD, for their guidance in conducting the urinary metabolite profiling. Editorial and technical assistance was provided by Quintiles Medical Communications, Parsippany, NJ.

\section{REFERENCES}

1 Gallwitz B. Saxagliptin, a dipeptidyl peptidase IV inhibitor for the treatment of type 2 diabetes. IDrugs 2008 ; 11: 906-17.

2 Wani JH, John-Kalarickal J, Fonseca VA. Dipeptidyl peptidase- 4 as a new target of action for type 2 diabetes mellitus: a systematic review. Cardiol Clin 2008; 26: 639-48.

3 Holst JJ, Deacon CF. Inhibition of the activity of dipeptidyl-peptidase IV as a treatment for type 2 diabetes. Diabetes 1998; 47: 1663-70.

4 Mclntosh CHS, Demuth H-U, Pospisilik JA, Pederson R. Dipeptidyl peptidase IV inhibitors: how do they work as new antidiabetic agents? Regul Pept 2005; 128: 159-65.

5 Onglyza [package insert]. Princeton, NJ: Bristol-Myers Squibb/Wilmington, DE: AstraZeneca; February 2011.

6 Boulton DW, Geraldes M. Safety, tolerability, pharmacokinetics and pharmacodynamics of once-daily oral doses of saxagliptin for 2 weeks in type 2 diabetic and healthy subjects [Abstract 606P]. Diabetes 2007; 56: (Suppl. 1): A161.

7 Patel CG, Boulton DW, Brenner E, Royzman K, Li L. Effect of ketoconazole on the pharmacokinetics of saxagliptin in healthy subjects [Abstract 89]. J Clin Pharmacol 2007; 47: 1183-211.

8 Girgis S, Patel CG, Li L, Gooding L, Frevert U, Whigan D, Boulton DW. Effect of diltiazem on the pharmacokinetics of saxagliptin in healthy subjects [Abstract 72]. J Clin Pharmacol 2007; 47: 1183-211.

9 Niemi M, Backman JT, Fromm MF, Neuvonen PJ, Kivistö KT. Pharmacokinetic interactions with rifampicin: clinical relevance. Clin Pharmacokinet 2003; 42: 819-50.

10 Backman JT, Olkkola KT, Neuvonen PJ. Rifampin drastically reduces plasma concentrations and effects of oral midazolam. Clin Pharmacol Ther 1996; 59: 7-13.
11 Gurley BJ, Swain A, Barone GW, Williams DK, Breen P, Yates CR, Stuart LB, Hubbard MA, Tong Y, Cheboyina S. Effect of goldenseal (Hydrastis canadensis) and kava kava (Piper methysticum) supplementation on digoxin pharmacokinetics in humans. Drug Metab Dispos 2007; 35: 240-5.

12 Giessmann T, Modess C, Hecker U, Zschiesche M, Dazert P, Kunert-Keil C, Warzok R, Engel G, Weitschies W, Cascorbi I, Kroemer HK, Siegmund W. CYP2D6 genotype and induction of intestinal drug transporters by rifampin predict presystemic clearance of carvedilol in healthy subjects. Clin Pharmacol Ther 2004; 75: 213-22.

13 Oswald S, Haenisch S, Fricke C, Sudhop T, Remmler C, Giessmann T, Jedlitschky G, Adam U, Dazert E, Warzok R, Wacke W, Cascorbi I, Kroemer HK, Weitschies W, von Bergmann K, Siegmund W. Intestinal expression of P-glycoprotein (ABCB1), multidrug resistance associated protein 2 (ABCC2), and uridine diphosphate-glucuronosyltransferase $1 \mathrm{~A} 1$ predicts the disposition and modulates the effects of the cholesterol absorption inhibitor ezetimibe in humans. Clin Pharmacol Ther 2006; 79: 206-17.

14 Ma X, Shah YM, Guo GL, Wang T, Krausz KW, Idle JR, Gonzalez FJ. Rifaximin is a gut-specific human pregnane $X$ receptor activator. J Pharmacol Exp Ther 2007; 322: 391-8.

15 Xiong H, Carr RA, Locke CS, Katz DA, Achari R, Doan TT, Wang P, Jankowski JR, Sleep DJ. Dual effects of rifampin on the pharmacokinetics of atrasentan. J Clin Pharmacol 2007; 47:423-9.

16 van Giersbergen PLM, Treiber A, Schneiter R, Dietrich H, Dingemanse J. Inhibitory and inductive effects of rifampin on the pharmacokinetics of bosentan in healthy subjects. Clin Pharmacol Ther 2007; 81:414-9.

17 Lau YY, Huang Y, Frassetto L, Benet LZ. Effect of OATP1B transporter inhibition on the pharmacokinetics of atorvastatin in healthy volunteers. Clin Pharmacol Ther 2007; 81: 194-204.

18 Vavricka SR, Van Montfoort J, Ha HR, Meier PJ, Fattinger K. Interactions of rifamycin SV and rifampicin with organic anion uptake systems of human liver. Hepatology 2002; 36 : 164-72.

19 Oswald S, Giessmann T, Luetjohann D, Wegner D, Rosskopf D, Weitschies W, Siegmund W. Disposition and sterol-lowering effect of ezetimibe are influenced by single-dose coadministration of rifampin, an inhibitor of multidrug transport proteins. Clin Pharmacol Ther 2006; 80: 477-85.

20 US Food and Drug Administration. Draft Guidance for Industry: Drug Interaction Studies - Study Design, Data Analysis, and Implications for Dosing and Labeling. Rockville, MD: US Food and Drug Administration, September 2006.

21 US Food and Drug Administration. Guidance for Industry: In Vivo Drug Metabolism/Drug Interaction Studies - Study Design, Data Analysis, and Recommendation for Dosing and Labeling. Rockville MD: US Food and Drug Administration, November 1999.

22 European Agency for the Evaluation of Medicinal Products. Note for Guidance on the Investigation of Drug Interactions. London: European Agency for the Evaluation of Medicinal Products, December 1997. 
B] CP v.v. Upreti et al.

23 Rifadin [package insert]. Bridgewater, NJ: sanofi-aventis U.S. LLC; March 2007.

24 US Food and Drug Administration. Code of federal regulations, title 21 - food and drugs: chapter I - Food and Drug Administration, Department of Health and Human Services. Part 56: institutional review boards [online]. Available at http://www.access.gpo.gov/nara/cfr/ waisidx_09/21cfr56_09.html (last accessed 12 October, 2009).

25 US Food and Drug Administration. Code of federal regulations, title 21 - food and drugs: chapter I - Food and
Drug Administration, Department of Health and Human Services. Part 50: protection of human subjects [online]. Available at http://www.access.gpo.gov/nara/cfr/ waisidx_09/21cfr50_09.html (last accessed 12 October, 2009).

26 Shah VP, Midha KK, Findlay JWA, Hill HM, Hulse JD, McGilveray IJ, McKay G, Miller KJ, Patnaik RN, Powell ML, Tonelli A, Viswanathan CT, Yacobi A. Bioanalytical method validation - a revisit with a decade of progress. Pharm Res 2000; 17: 1551-7. 